\title{
Validación del Cuestionario de Actitudes Socioculturales sobre la Apariencia (SATAQ-4) en población colombiana
}

\author{
Martha Juliana Villegas Moreno; Constanza Londoño Pérez; Carlos Pardo Adames
}

Cómo citar este artículo:

Villegas Moreno, M., J., Londoño Pérez, C., \& Pardo Adames, C. (2021). Validación del Cuestionario de Actitudes Socioculturales sobre la Apariencia (SATAQ-4) en población colombiana. Acta Colombiana de Psicología, 24(1), 86-95. https://www.doi.org/10.14718/ACP.2021.24.1.8

Recibido, septiembre 27/2019; Concepto de evaluación, julio 21/2020; Aceptado, septiembre 25/2020

\author{
Martha Juliana Villegas Moreno ${ }^{1}$ \\ ORCID: https://orcid.org/0000-0002-9059-0600 \\ Universidad Católica de Pereira, Pereira, Colombia. \\ Constanza Londoño Pérez \\ ORCID: https://orcid.org/0000-0003-3273-3658 \\ Universidad Católica de Colombia, Bogotá, Colombia. \\ Carlos Pardo Adames \\ ORCID: https://orcid.org/0000-0001-6773-2781 \\ Universidad Católica de Colombia, Bogotá, Colombia.
}

\begin{abstract}
Resumen
El deseo de cumplir estándares modernos estéticos corporales no alcanzables afecta la salud mental, debido a que las personas internalizan los ideales de belleza y aceptan las presiones sociales acerca de la apariencia corporal incluso por encima de su bienestar. El objetivo del presente estudio instrumental fue evaluar las propiedades psicométricas del Cuestionario de actitudes socioculturales sobre la apariencia (SATAQ-4) en población colombiana, para lo cual se utilizó una muestra estratificada no aleatorizada conformada por 214 personas - 138 mujeres y 76 hombres — , con un promedio de edad de 25.13 años $(D E=7.66)$. Se retomó la versión en español del instrumento validado en Argentina, que fue evaluada por jueces expertos para realizar la adaptación lingüística y que se aplicó a una muestra piloto antes de la aplicación definitiva, hecha bajo las normas éticas exigidas. Se realizó el análisis de las condiciones psicométricas desde la teoría de respuesta al ítem, mediante el modelo Rasch, y por medio del software wINSTEPS. Los resultados indican que la versión en español cumple las condiciones psicométricas adecuadas en las cinco dimensiones que componen el instrumento (.72-.94); y que el grado de dificultad y de habilidad alcanzan valores de ajuste apropiados, lo que indica que el cuestionario cuenta con adecuadas condiciones psicométricas y mantiene su estructura interna original. En conclusión, la versión final de esta adaptación del SATAQ-4 puede ser utilizada en población de similar procedencia, a fin de evaluar el efecto de los aspectos socioculturales en la satisfacción con la imagen corporal.

Palabras clave: imagen corporal, influencias socioculturales, propiedades psicométricas, trastornos de conducta alimentaria.
\end{abstract}

\section{Validation of the Sociocultural Attitudes Questionnaire on Appearance (SATAQ-4) in the Colombian population}

\begin{abstract}
The desire to meet modern aesthetic but unattainable body standards affects mental health, because people internalize the ideals of beauty and accept social pressures about body appearance even beyond their well-being. The objective of this instrumental study was to evaluate the psychometric properties of the Sociocultural Attitude Questionnaire on Appearance (SATAQ-4) in the Colombian population, using a non-randomized stratified sample of 214 people, 138 women and 76 men, with an average age of 25.13 years $(\mathrm{SD}=7.66)$. The Spanish version of the instrument validated in Argentina was used again, which was evaluated by expert judges to perform the linguistic adaptation and apply it to a pilot sample before the final
\end{abstract}

Avenida de las Américas n. ํ 49-95, Pereira, Colombia. Tel.: (57) (6) 3124000. Correo electrónico: martha.villegas@ucp.edu.co 
application, made under the required ethical standards. The analysis of the psychometric conditions was carried out from the Item Response Theory, by using the Rasch model, and through the WINSTEPS software. The results indicate that the Spanish version fulfills the adequate psychometric conditions in the five dimensions that make up the instrument (.72-.94); and that the degree of difficulty and ability reach appropriate adjustment values, which indicates that the questionnaire has adequate psychometric conditions and maintains its original internal structure. In conclusion, the final version of this adaptation of SATAQ-4 can be used in a population of similar origin, in order to evaluate the effect of sociocultural aspects on satisfaction with body image.

Keywords: body image, sociocultural influences, psychometric properties, eating disorders.

\section{Introducción}

Los trastornos de la conducta alimentaria (TCA) se ubican entre las enfermedades mentales de mayor importancia en la población de niños y adolescentes, debido al riesgo para la salud física y mental que representan y al efecto negativo que tienen sobre la calidad de vida de estos (Bautista \& Serra, 2015; Bryant-Waugh, 2019; Buelow, 2020; Drieberg et al., 2019; Guarín et al., 2013; Linardon et al., 2019; Ochoa et al., 2013; OMs, 2018; Pérez et al., 2018; Solís et al., 2019; Williams-Kerver \& Crowther, 2020).

Recientemente, la Asociación Nacional de Trastornos de la Alimentación (NEDA, 2018) planteó que entre el $0.9 \% \mathrm{y}$ el $2.0 \%$ de las mujeres y entre el $0.1 \%$ y el $0.3 \%$ de los hombres desarrollarán anorexia en su vida, mientras que entre el $1.1 \%$ y el $4.6 \%$ de las mujeres y entre el $0.1 \%$ y el $0.5 \%$ de los hombres desarrollarán bulimia. De hecho, se sabe que en México, en mujeres adolescentes (12-17 años) residentes en zona urbana, se calculó una prevalencia de $0.05 \%$ de anorexia, $1.0 \%$ de bulimia nerviosa, y $1.4 \%$ de trastorno por atracón (TPA) (Meléndez et al., 2017); mientras que en Colombia, Fajardo et al. (2017) encontraron que el riesgo de que estudiantes de secundaria presenten TCA es de $0.3 \%$, y Pérez et al. (2015) afirman que el riesgo de bulimia es de $0.4 \%$ en adolescentes mayores de 16 años.

Las causas de los TCA son múltiples y están relacionadas con la insatisfacción, distorsión y excesiva preocupación por la imagen corporal (Bornioli et al., 2019; Corno et al., 2018; Cuquejo et al., 2017; Plumed et al., 2019; Salmeron et al., 2017; Sharpe et al., 2018); y se ha encontrado que la satisfacción con la imagen corporal está influenciada por factores socioculturales, entre los que se incluyen los estándares de belleza establecidos respecto a la figura y arreglo corporal (Gonçalves-Câmara \& BedinTomasi, 2015; Ralph-Nearman \& Filik, 2020; Schnettler et al., 2018; Vaquero-Cristóbal et al., 2013).

Al respecto, Behar (2010) expone que la sociedad occidental ha promovido valores, creencias y cánones estéticos que han dado origen a estereotipos corporales que establecen un arquetipo de belleza centrado en el culto al cuerpo joven y delgado, especialmente para las mujeres, y de musculatura sin grasa para los hombres (Boon et al., 2017; Eik-Nes et al., 2018; Prnjak et al., 2019).

Específicamente, Ruíz et al. (2010) consideran influencias culturales, en el modelo estético corporal, a todas aquellas prácticas sociales y creencias difundidas en revistas de circulación masiva, televisión, productos adelgazantes y redes sociales, que por razones estéticas promueven el adelgazamiento y la delgadez extrema (Cortez et al., 2016; Forney et al., 2019; Gonçalves \& Martínez, 2014; JiménezFlores et al., 2017; Rodgers, Lowy et al., 2016; Shahyad et al., 2018; Uchôa et al., 2019); prácticas que llegan a generar presión sobre las personas para alcanzar el ideal estético.

Como se demuestra en diversas investigaciones, la interiorización de este modelo produce tanto insatisfacción con la imagen corporal como la aparición de conductas de riesgo de trastorno alimentario, puesto que el sujeto tiende a evaluar la distancia entre las características del cuerpo ideal y el real, hecho que afecta su estado de ánimo y disminuye su autoestima (Al-Kloub et al., 2018; Carrard et al., 2020; Cortez et al., 2016; Leins et al., 2018; Murawski et al., 2015; Rheanna et al., 2013).

Ahora, existen diversos instrumentos que evalúan aspectos relacionados con la imagen corporal; sin embargo, la mayoría centran la atención en la forma en que las personas perciben su cuerpo y el grado de satisfacción que reportan respecto a la apariencia socialmente reflejada, pero no abordan los aspectos culturales ni su función. Entre ellos, están las diferentes versiones del Cuestionario de influencias del modelo estético corporal (CIMEC), de Vázquez et al. (2000), el Instrumento para la percepción de la imagen corporal (IPIC), de Campayo et al. (2003), y el Cuestionario de Imagen corporal para personas en situación de discapacidad, de Botero-Soto y Londoño-Pérez (2015).

Por otra parte, se encontró que el Cuestionario de actitudes socioculturales sobre la apariencia (SATAQ-4), una revisión del SATAQ-3 - que no contaba con una subescala que midiera internalización del ideal muscular-, que tiene como propósito proporcionar una evaluación más completa de las presiones sociales, pues incluye elementos 
relacionados con la familia, los compañeros y los medios de comunicación con el fin de obtener una medida de internalización del ideal de delgadez y muscular más enfocada, así como ajustar sistemáticamente la confiabilidad y la validez convergente de las puntuaciones en la nueva medida en muestras femeninas, masculinas, e interculturalmente (Schaefer et al., 2015; Thompson et al., 2004).

La primera versión del SATAQ-R fue desarrollada por Cusumano y Thompson (1997) en Estados Unidos, mientras que el ajuste de las versiones SATAQ-3 y SATAQ-4 fue realizado por Schaefer et al. (2012). La última adaptación ha sido validada en países europeos - Italia, Inglaterra, España, Francia - asiáticos - Japón - y en el continente australiano, estudios en los que se conservaron los adecuados índices de consistencia interna y se mantuvo su estructura factorial original (Llorente et al., 2015; Rodgers, Schaefer et al., 2016; Schaefer et al., 2012; Schaefer et al., 2015; Yamamiya et al., 2016; Yamamiya et al., 2019) (véase Tabla 1); condición que, aunque facilita su uso en diferentes poblaciones de Latinoamérica, no permite valorar el aspecto en Colombia.

Tabla 1.

Validaciones disponibles del SATAQ-4

\begin{tabular}{lccc}
\hline $\begin{array}{l}\text { Autores } \\
\text { del estudio }\end{array}$ & $\begin{array}{c}\text { País/población } \\
\text { de validación }\end{array}$ & $\begin{array}{c}\text { Propiedades psicométricas } \\
\text { Confiabili- } \\
\text { dad } \alpha\end{array}$ & $\begin{array}{c}\text { Estructura } \\
\text { factorial }\end{array}$ \\
\hline $\begin{array}{l}\text { Schaefer } \\
\text { et al. (2012) }\end{array}$ & $\begin{array}{c}\text { Italia, Inglaterra } \\
\text { y Australia }\end{array}$ & .83 & 5 subescalas \\
$\begin{array}{l}\text { Llorente } \\
\text { et al. (2015) }\end{array}$ & España & .89 & 5 subescalas \\
$\begin{array}{l}\text { Yamamiya } \\
\text { et al. (2016) }\end{array}$ & Japón & .89 & 5 subescalas \\
$\begin{array}{l}\text { Yamamiya } \\
\text { et al. (2019) }\end{array}$ & Japón & .90 & 5 subescalas \\
$\begin{array}{l}\text { Rodgers, } \\
\text { Schaefer } \\
\text { et al. (2016) }\end{array}$ & Francia & .83 & 5 subescalas \\
\hline
\end{tabular}

Teniendo lo anterior en cuenta, es importante tener instrumentos válidos y confiables para evaluar actitudes psicológicas, culturales y sociales respecto a la apariencia corporal, pues estas han sido relacionadas con la aparición de TCA, depresión, ansiedad y trastorno dismórfico corporal; condiciones psicopatológicas cuya incidencia y prevalencia han aumentado durante la última década tanto en la población latinoamericana como en Colombia. Por tanto, es preciso contar con un instrumento psicométricamente válido que permita ejecutar a tiempo acciones de prevención y de acompañamiento con esta población. Por ello, el objetivo del presente estudio instrumental fue evaluar las propiedades psicométricas del Cuestionario de actitudes socioculturales sobre la apariencia (SATAQ-4) en una muestra de población colombiana.

\section{Método}

Tipo de estudio

Se llevó a cabo un estudio psicométrico de tipo instrumental, dado que apunta al desarrollo de pruebas, así como a su diseño y respectiva adaptación (Montero \& León, 2002; Paniagua, 2015).

\section{Participantes}

Se utilizó una muestra estratificada no aleatorizada conformada por 214 personas —estudiantes y personal administrativo de dos instituciones de educación superior-, 138 mujeres $(64.5 \%)$ y 76 hombres $(35.5 \%)$, con un promedio de edad de 25.13 años y una desviación estándar de 7.66. De acuerdo con la recomendación de Nunnally y Bernstein (1978), se incluyeron 10 personas por ítem del cuestionario.

\section{Instrumento}

El SATAQ-4 es una prueba de autoinforme compuesta por 22 reactivos que evalúan las actitudes socioculturales hacia la apariencia, y está conformada por cinco subescalas que miden: (a) la internalización del ideal muscular (5 ítems), (b) la internalización del ideal de delgadez (5 ítems), (c) las presiones de los familiares (4 ítems), (d) las presiones de los pares (4 ítems), y (e) las presiones de los medios de comunicación (4 ítems) (véase Tabla 2). Además, la escala de respuesta del instrumento es de tipo Likert, donde 1 corresponde a "completamente en desacuerdo", y 5, a "completamente de acuerdo". La calificación del cuestionario es: a mayor puntaje, mayor internalización e influencia del modelo estético corporal (Rodgers, Schaefer et al., 2016).

\section{Procedimiento}

En primer lugar, el instrumento traducido al idioma español se evaluó con el juicio de cuatro investigadores expertos disciplinares en el área, acerca de la suficiencia, claridad, coherencia, relevancia y utilidad de los ítems. Para medir el nivel de concordancia entre los jueces, se hizo el cálculo del coeficiente $\mathrm{W}$ de Kendall, y la matriz utilizada para ello fue retomada de Escobar-Pérez y Cuervo-Martínez (2008). Una vez recibidas las valoraciones, se llevó a cabo el proceso de adaptación lingüística, lo que implicó buscar conceptos equivalentes en el contexto sociocultural, con el cuidado de preservar el significado original de los términos 
Tabla 2.

Composición del SATAQ-4 por subescalas y reactivos

Componentes

Internalización muscular

1. Es importante para mí parecer atlético/a.

2. Pienso mucho en parecer musculoso/a.

6. Paso mucho tiempo haciendo cosas para parecer más atlético/a.

7. Pienso mucho en parecer atlético/a.

10. Paso mucho tiempo haciendo cosas para parecer más musculoso/a.

Internalización de la delgadez

3. Quiero que mi cuerpo parezca muy delgado.

4. Quiero que mi cuerpo parezca que tiene poca grasa.

5. Pienso mucho en parecer delgado/a.

8. Quiero que mi cuerpo parezca muy magro (con muy poca grasa).

9. Pienso mucho en tener muy poca grasa corporal.

\section{Presiones familiares}

11. Siento presión de los miembros de mi familia para parecer más delgado/a.

12. Siento presión de los miembros de mi familia para mejorar mi apariencia.

13. Los miembros de mi familia me animan a reducir mi nivel de grasa corporal.

14. Los miembros de mi familia me animan a ponerme en mejor forma.

$$
\text { Presiones por parte de los pares }
$$

15. Mis compañeros/as me animan a adelgazar.

16. Siento presión de mis compañeros/as para mejorar mi apariencia.

17. Siento presión de mis compañeros/as para parecer en mejor forma.

18. Siento presión de mis compañeros/as para reducir mi nivel de grasa corporal.

$$
\text { Presiones de los medios de comunicación }
$$

19. Siento presión de los medios para parecer en mejor forma.

20. Siento presión de los medios para parecer más delgado/a.

21. Siento presión de los medios para mejorar mi apariencia.

22. Siento presión de los medios para reducir mi nivel de grasa corporal.

modificados. Posteriormente, se aplicó el instrumento en físico de manera individual; y finalmente, se realizaron los respectivos análisis de confiabilidad y de otras condiciones psicométricas para dar evidencia del funcionamiento adecuado del cuestionario.

\section{Consideraciones éticas}

Los participantes, de manera voluntaria, diligenciaron el consentimiento informado, en el cual se exponía el objetivo del estudio y el profesional responsable de él, las condiciones de confidencialidad, el anonimato de las respuestas, el uso de la información, los términos de publicación de resultados, y los posibles riesgos para la persona, pues se les advirtió a los participantes que si el resultado de su prueba indicaba algún riesgo para su salud mental, serían remitidos al centro de salud para ser atendidos debidamente - condición que no se evidenció dentro del proceso de recolección de información-. Es importante señalar que el estudio fue aprobado por el comité de ética institucional de la Universidad Católica de Colombia, y que su nivel de riesgo era "muy bajo".

\section{Resultados}

\section{Validez de contenido}

Como resultados, el nivel de concordancia del juicio de expertos fue alto (Coeficiente $\mathrm{W}$ de Kendall $=.4$ ) y significativo $(p=.039)$, los que denota que los conceptos emitidos fueron favorables y con tendencia media a afinidad. Específicamente, las principales indicaciones estuvieron relacionadas con la redacción para dar mayor claridad a afirmaciones, como "mejorar la apariencia" y "parecer de mejor forma", por lo que esas frases se modificaron por 
"apariencia física". Respecto del uso del término "magro", dado que no es frecuente en Colombia, se decidió cambiarlo por la expresión "poca grasa". Una vez ajustados los reactivos, se hizo una prueba piloto con 15 participantes, quienes reportaron un alto nivel de comprensión de los ítems. A partir de la consideración unificada de los participantes de la prueba piloto, se eliminó el reactivo 8: "Pienso mucho en tener muy poca grasa corporal", pues consideraron que la pregunta 5: "Pienso mucho en parecer delgado/a" cubría estos dos aspectos — delgadez y peso-; decisión que no afectó la validez de constructo.

\section{Propiedades psicométricas de la adaptación}

Para evaluar la confiabilidad, validez, estructura, discriminación y determinación de puntos de corte, se usó el modelo Rasch; mientras que, para evaluar el funcionamiento diferencial de ítems, se calculó el índice Mantel Haenszel (мн). Por otra parte, la validez concurrente se evaluó a través del análisis de covariación y los índices de correlación de Pearson -índice de significación $\geq .05-$. En general, la versión adaptada del instrumento obtuvo un nivel de confiabilidad alto, lo cual se refleja tanto en el índice de separación (2.63) como desde la teoría tradicional, a partir del alfa de Cronbach $(\alpha=.87)$ (véase Tabla 3 ).

Aunque algunos ítems presentaron en el infit valores próximos al límite inferior del intervalo deseable (1-1.3), al analizar los puntajes del outfit se encuentra que todos los ítems presentaron puntajes menores a 2.0, tal como se esperaba. Es importante anotar que, para aceptar la conservación de los ítems, se debe cumplir al menos con uno de los criterios establecidos respecto a los valores relacionados con el índice de separación (véase Tabla 4).

Sumado a esto, se encuentra que las opciones de respuesta ofrecidas por los ítems tienen probabilidades de elección equiparables, aunque se denota una tendencia de elección de las opciones de respuesta que indican alta frecuencia. Adicionalmente, la distribución en el diagrama de los ítems y los participantes se encontraban entre 1 y -2 logits, lo que indica alta comprensión de los ítems y alta correspondencia entre la comprensión y el nivel de presentación del constructo (véase Figura 1).

\section{Discusión}

Los resultados del presente estudio permiten afirmar que en la muestra colombiana se confirman las cinco dimensiones - ideal de delgadez, ideal muscular, presiones de la familia, presiones de los pares, y presiones de los medios de comunicación - que hacen parte del SATAQ-4, lo cual indica que el instrumento evalúa adecuadamente el constructo en esta población. Por ello, se confirman las condiciones de calificación y estructura de la prueba (Llorente et al., 2015; Schaefer et al., 2015).

Asimismo, la prueba cumplió el criterio de independencia local de respuestas, puesto que no se presenta un patrón general de elección de opciones, lo que denota que las opciones de respuesta elegidas por los participantes no se da por mutua influencia (Linacre, 2002). Todas las opciones de respuesta ofrecidas mostraban la misma probabilidad de ser escogidas.

Además, cabe anotar que el ajuste de los ítems reflejado en los indicadores de los índices de separación infit y outfit muestran un nivel apropiado, lo que indica que los reactivos fueron respondidos uno a uno, de manera independiente, y no como un patrón; de hecho, el DIF permite identificar que no hay un funcionamiento específico de los ítems dependiente del sexo o la edad (Hambleton \& Jones, 1993).

Por otra parte, los índices de ajuste de los ítems permiten afirmar que estos aportan de forma adecuada a la medición de influencias socioculturales, en tanto el comportamiento de los datos representa la relación entre los valores del ajuste próximo (infit) y del ajuste lejano (outfit) (Pardo \& Rocha, 2010); y, por ende, puede inferirse que en el caso

Tabla 3.

Separación, ajuste y consistencia interna del SATAQ-4

\begin{tabular}{|c|c|c|c|c|c|c|}
\hline & $\begin{array}{l}\text { Puntaje } \\
\text { total }\end{array}$ & Ítems contados & Error del modelo & $\begin{array}{c}\text { Infit } \\
\text { MNSQ }\end{array}$ & $\begin{array}{l}\text { Outfit } \\
\text { MNSQ }\end{array}$ & \\
\hline Media & 49.8 & 21 & 0.23 & 1.01 & 1.00 & \\
\hline$D T$ & 0.0 & 0.0 & 0.01 & 0.04 & 0.04 & \\
\hline Máx. & 81 & 21 & 3.01 & 3.01 & 3.03 & \\
\hline Mín. & 22 & 21 & 0.20 & 0.20 & 0.20 & \\
\hline RSME Real & 0.27 & & Separación & 2.40 & Confiabilidad & .85 \\
\hline RSME Modelo & 0.25 & & Separación & 2.63 & Confiabilidad & .87 \\
\hline De participantes & 0.05 & & & & & \\
\hline
\end{tabular}


Tabla 4.

Indicadores psicométricos de los items

\begin{tabular}{|c|c|c|c|c|c|c|c|c|c|}
\hline \multirow{2}{*}{ Ítem } & \multirow{2}{*}{$\mathrm{PT}$} & \multirow{2}{*}{$\mathrm{X}$} & \multirow{2}{*}{$\mathrm{XM}$} & \multirow{2}{*}{$\begin{array}{l}\text { Infit } \\
\text { MNSQ }\end{array}$} & \multirow{2}{*}{$\begin{array}{l}\text { Outfit } \\
\text { MNSQ }\end{array}$} & \multicolumn{2}{|c|}{ PTM MEDIDA } & \multirow{2}{*}{$\begin{array}{c}\text { OBS } \\
\text { EXAC } \%\end{array}$} & \multirow{2}{*}{$\begin{array}{l}\text { MATCH } \\
\text { EXO \% }\end{array}$} \\
\hline & & & & & & COR & EXP & & \\
\hline 1 & 684 & -.83 & .07 & 0.98 & 1.09 & .46 & .58 & 32.9 & 35.6 \\
\hline 2 & 463 & .15 & .07 & 1.20 & 1.23 & .34 & .48 & 34.3 & 36.3 \\
\hline 3 & 543 & -.21 & .07 & 0.80 & 0.92 & .50 & .52 & 40 & 34.2 \\
\hline 4 & 637 & -.62 & .07 & 0.81 & 0.78 & .61 & .57 & 43.8 & 34.5 \\
\hline 5 & 557 & -.27 & .07 & 0.83 & 0.82 & .56 & .53 & 41.0 & 33.9 \\
\hline 6 & 511 & -.07 & .07 & 1.04 & 1.01 & .44 & .51 & 33.3 & 34.0 \\
\hline 7 & 538 & -.19 & .07 & 0.92 & 1.00 & .49 & .52 & 35.7 & 34.2 \\
\hline 8 & 537 & -.19 & .07 & 0.76 & 0.74 & .58 & .52 & 41.0 & 33.9 \\
\hline 9 & 447 & .23 & .07 & 1.14 & 1.09 & .37 & .47 & 36.7 & 37.1 \\
\hline 10 & 358 & .73 & .08 & 1.15 & 1.00 & .41 & .39 & 51.4 & 46.8 \\
\hline 11 & 408 & .43 & .07 & 1.32 & 1.31 & .39 & .44 & 37.6 & 40.9 \\
\hline 12 & 451 & .21 & .07 & 1.00 & 1.01 & .52 & .47 & 34.3 & 37.0 \\
\hline 13 & 560 & -.29 & .07 & 1.17 & 1.21 & .50 & .53 & 30.0 & 33.9 \\
\hline 14 & 395 & .50 & .08 & 0.93 & 1.10 & .45 & .42 & 46.7 & 41.2 \\
\hline 15 & 376 & .61 & .08 & 0.87 & 0.78 & .49 & .41 & 51.0 & 43.2 \\
\hline 16 & 330 & .93 & .09 & 0.72 & 0.58 & .49 & .35 & 62.4 & 54.7 \\
\hline 17 & 377 & .61 & .08 & 0.91 & 0.88 & .46 & .41 & 50.5 & 43.2 \\
\hline 18 & 616 & -.53 & .07 & 1.24 & 1.18 & .56 & .56 & 29.5 & 34.4 \\
\hline 19 & 571 & -.33 & .07 & 1.16 & 1.09 & .57 & .54 & 26.2 & 34.1 \\
\hline 20 & 630 & -.59 & .07 & 1.19 & 1.15 & .57 & .56 & 30.0 & 34.5 \\
\hline 21 & 557 & -.27 & .07 & 1.09 & 1.01 & .59 & .53 & 31.9 & 33.9 \\
\hline$X$ & 502.2 & .00 & .07 & 1.01 & 1.00 & & & 39.0 & 37.7 \\
\hline$D T$ & 99.6 & .48 & .01 & 0.17 & 0.18 & & & 8.9 & 5.4 \\
\hline
\end{tabular}

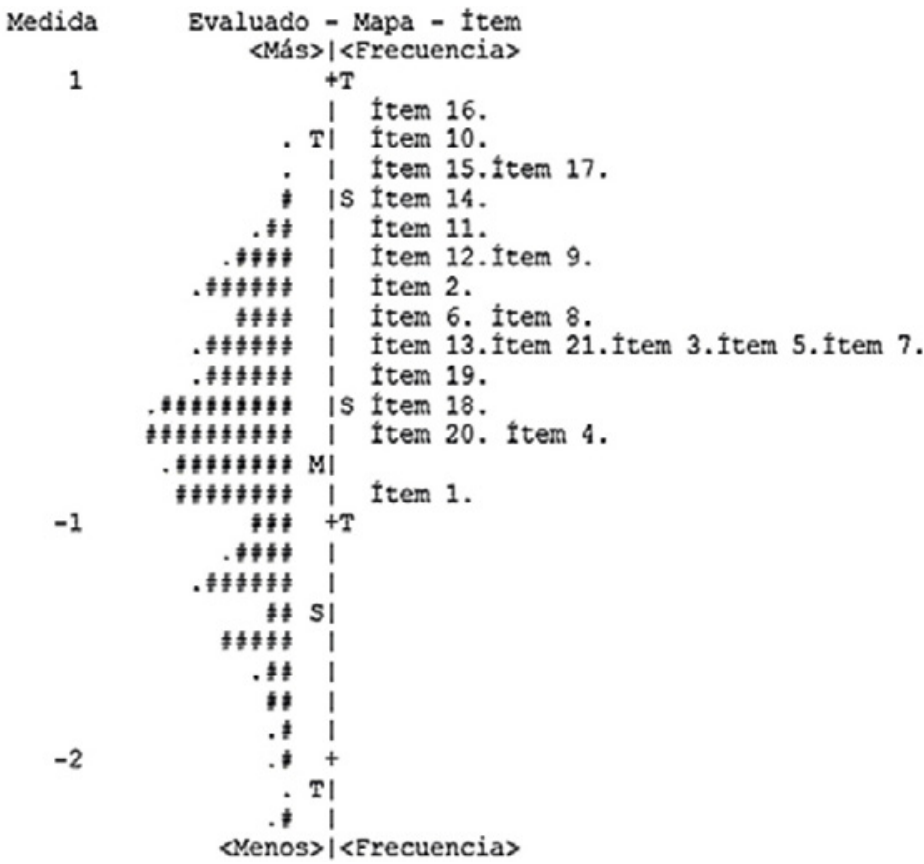

Figura 1 .

Mapa de distribución de dificultades y habilidades 
particular del SATAQ-4 hay cumplimiento de los supuestos de dicho modelo.

Cabe anotar que hacer el análisis desde la perspectiva de la teoría de respuesta al ítem permite mejorar la medición de los procesos psicológicos, puesto que, al ser los ítems la unidad de análisis, se facilita el uso de indicadores no dependientes de la muestra, lo que ofrece un análisis funcional entre el rasgo evaluado de quien responde y el ítem usado para evaluarlo, además de que se aportan datos específicos acerca del ajuste del modelo que sustenta al concepto base (Fischer \& Prieto, 2016; Gâmez, 2015; Londoño et al., 2019; Pedrosa et al., 2013).

Adicionalmente, este proceso de validación del SATQ-4 permite confirmar qué personas muestran una tendencia hacia una fuerte influencia de factores socioculturales en la construcción y satisfacción con la imagen corporal, tal como lo indica un número importante de estudios previos (Amaya-Hernández et al., 2017; Cortez et al., 2016; EscolarLlamazares et al., 2017; Pejenaute \& Labari, 2018).

Ahora bien, es importante señalar que, a pesar de la eliminación del ítem 8 de internalización de delgadez, esto no afectó las condiciones psicométricas generales del cuestionario; y que la adaptación del SATAQ-4 para población colombiana muestra el buen funcionamiento de la prueba, porque no hubo reporte de dificultades en la claridad, comprensión o elección de opción de respuesta a los ítems.

Finalmente, es necesario indicar que validar un instrumento que mide la influencia sociocultural sobre la apariencia corporal en población colombiana permite identificar el grado en que las personas internalizan los ideales estéticos corporales actuales, así como el nivel de influencia familiar, social y de los medios de comunicación; factores que determinan en gran medida la insatisfacción con la imagen corporal, la que a su vez se encuentra dentro de las variables etiológicas, tanto de los trastornos de conducta alimentaria, como de los dismórficos corporales, fenómenos que no solo presentan altas prevalencias, sino que, en muchas ocasiones, llevan a las personas a someterse a tratamientos estéticos que ponen en peligro su salud $y$, en la peor de las circunstancias, su vida.

\section{Limitaciones y futuras direcciones}

Si bien los resultados obtenidos en el estudio indican que es posible el uso del instrumento en población colombiana, es importante anotar que la muestra de este estudio estaba localizada en una zona del país —el Eje Cafetero colombiano-, por lo cual se recomienda confirmar los resultados en otras regiones, e incluso ampliar los rangos de edad de los participantes.
Nuevas investigaciones pueden girar en torno a la validación del SATAQ-4 con grupos poblacionales con características específicas, como personas diagnosticadas con algún tipo de trastorno de conducta alimentaria, trastorno dismórfico corporal, o de manera diferencial por edades, sexo y género.

\section{Referencias}

Al-Kloub, M. I., Al-Khawaldeh, O. A., ALBashtawy, M., Batiha, A. M., \& Al-Haliq, M. (2018). Disordered eating in Jordanian adolescents. International Journal of Nursing Practice, 25(10), e12694. https://doi.org/10.1111/ijn.12694

Amaya-Hernández, A., Álvarez-Rayón, G., Ortega-Luyando, M., \& Mancilla-Díaz, J. M. (2017). Peer influence in preadolescents and adolescents: A predictor of body dissatisfaction and disordered eating behaviors. Revista Mexicana de Trastornos Alimentarios, 8(1), 31-39. https://doi. org/10.1016/j.rmta.2016.12.001

Asociación Nacional de Trastornos de Alimentación (NEDA). (2018). Statistics \& Research on Eating Disorders. https://www.nationaleatingdisorders.org/ statistics-research-eating-disorders

Bautista C. I., \& Serra M. L. (2015). Prevalencia de los trastornos de la conducta alimentaria en adolescentes de Gran Canaria. Nutrición Hospitalaria, 31(5), 2283-2288. https:// doi.org/10.3305/nh.2015.31.5.8583

Behar, R. (2010). La construcción cultural del cuerpo: El paradigma de los trastornos de la conducta alimentaria. Revista Chilena de Neuro-Psiquiatria, 48(4), 319-334. http:// dx.doi.org/10.4067/S0717-92272010000500007

Boon, E., Zainal, K. A., \& Touyz, S. W. (2017). Perceptions of eating disorder diagnoses and body image issues in four male cases in Singapore. Journal of Eating Disorders, 5, 1-6. https://doi.org/10.1186/s40337-017-0159-X

Bornioli, A., Lewis-Smith, H., Smith, A., Slater, A., \& Bray, I. (2019). Adolescent body dissatisfaction and disordered eating: Predictors of later risky health behaviours. Social Science \& Medicine, 238, 112458. https://doi.org/10.1016/j. socscimed.2019.112458

Botero-Soto, P. A., \& Londoño-Pérez, C. (2015). Diseño y validación de un Cuestionario de Imagen Corporal para Personas en Situación de Discapacidad Física. Revista Colombiana de Psicología, 24(1), 219-233. http://dx.doi. org/10.15446/rcp.v24n1.45644

Bryant-Waugh, R. (2019). Feeding and Eating Disorders in Children. The Psychiatric Clinics of North America, 42(1), 157-167. https://doi.org/10.1016/j.psc.2018.10.005

Buelow, M. T. (2020). Disordered eating behaviors: anorexia, bulimia, binge eating, and obesity. En M. Buelow (Ed.), Risky Decision Making in Psychological Disorders (pp. 135-148). Academic Press. https://doi.org/10.1016/ B978-0-12-815002-3.00007-3 
Campayo, R., Fernández, L., Cano, T., \& Martínez-Sánchez, F. (2003). Adaptación española de la escala de evaluación de la imagen corporal de Gardner en pacientes con trastornos de la conducta alimentaria. Actas Españolas de Psiquiatría, 31(2), 59-64. https://psycnet.apa.org/ record/2003-99362-002

Carrard, I., Rothen, S., \& Rodgers, R. F. (2020). Body image and disordered eating in older women: A Tripartite Sociocultural model. Eating Behaviors, 38, 101412-101416. https://doi. org/10.1016/j.eatbeh.2020.101412

Corno, G., Serino, S., Cipresso, P., Baños, R. M., \& Riva, G. (2018). Assessing the relationship between attitudinal and perceptual component of body image disturbance using virtual reality. Cyberpsychology, Behavior, and Social Networking, 21(11), 679-686. https://doi.org/10.1089/ cyber.2018.0340

Cortez, D., Gallegos, M., Jiménez, T., Martínez, P., Saravia, S., Cruzat-Mandich, C., Díaz-Castrillón, F., Behar, R., \& Arancibia, M. (2016). Influence of sociocultural factors on body image from the perspective of adolescent girls. Revista Mexicana de Trastornos Alimentarios, 7(2), 116124. https://doi.org/10.1016/j.rmta.2016.05.001

Cuquejo, L. M., Aguiar, C., Domínguez, G. C., \& Hermosilla, A. T. (2017). ¿Trastornos de la conducta alimentaria en adolescentes: una patología en auge? Pediatría (Asunción), 44(1), 37-42. https://www.revistaspp.org/index.php/ pediatria/article/view/151

Cusumano, D., \& Thompson J. K. (1997). Body image and body shape ideals in magazines: Exposure, awareness and internalization. Sex Roles, 37(9/10), 701-721. https://doi. org/10.1007/BF02936336

Drieberg, H., McEvoy, P. M., Hoiles, K. J., Shu, C. Y., \& Egan, S. J. (2019). An examination of direct, indirect and reciprocal relationships between perfectionism, eating disorder symptoms, anxiety, and depression in children and adolescents with eating disorders. Eating Behaviors, 32, 53-59. https://doi.org/10.1016/j.eatbeh.2018.12.002

Eik-Nes, T. T., Austin, S. B., Blashill, A. J., Murray, S. B., \& Calzo, J. P. (2018). Prospective health associations of drive for muscularity in young adult males. International Journal of Eating Disorders, 51(10), 1185-1193. https://doi. org/10.1002/eat.22943

Escobar-Pérez, J., \& Cuervo-Martínez, Á. (2008). Validez de contenido y juicio de expertos: una aproximación a su utilización. Avances en Medición, 6(1), 27-36. http://www. humanas.unal.edu.co/psicometria/files/7113/8574/5708/ Articulo3_Juicio_de_expertos_27-36.pdf

Escolar-Llamazares, M. C., Martín, M. Á. Alonso, M. Y., Gómez, M. B., Val, E. M., \& Ortega, F. L. (2017). Risk factors of eating disorders in university students: Estimation of vulnerability by sex and age. Revista Mexicana de Trastornos Alimentarios, 8(2), 105-112. https://doi. org/10.1016/j.rmta.2017.05.003
Fajardo, E., Méndez, C., \& Jauregui, A. (2017). Prevalencia del riesgo de trastornos de la conducta alimentaria en una población de estudiantes de secundaria, Bogotá-Colombia. Revista Médica, 25(1), 46-57. https://doi.org/10.18359/ rmed.2917

Fischer, R., \& Prieto, G. A. (2016). Análisis del Test de Actitudes Alimentarias (EAT-26) con un modelo tipo Rasch en una muestra de adolescentes paraguayos. Cuadernos Hispanoamericanos de Psicología, 16(1), 5-16. https://dialnet.unirioja.es/servlet/articulo?codigo $=5855294$

Forney, K. J., Schwendler, T., \& Ward, R. M. (2019). Examining similarities in eating pathology, negative affect, and perfectionism among peers: A social network analysis. Appetite, 137, 236-243. https://doi.org/10.1016/j.appet.2019.03.013

Gámez, C. A. (2015). Diseño construcción y validación de un instrumento que evalúa clima organizacional en empresas colombianas desde la teoría de respuesta al item. Acta Colombiana de Psicología, (11), 97-113. https://actacolom bianapsicologia.ucatolica.edu.co/article/view/482

Gonçalves-Câmara, S., \& Bedin-Tomasi, L. M. (2015). Bienestar, salud e imagen corporal de adolescentes brasileros: la importancia de los contextos familiares, de amistad y escolar. Universitas psychologica, 14(4), 1399-1409. http:// dx.doi.org/10.11144/Javeriana.up14-4.bsic

Gonçalves, V. O., \& Martínez, J. P. (2014). Imagen corporal y percepción de la influencia de los medios de comunicación: diferencias de género en una muestra de adolescentes. Revista Inter Ação, 39(3), 461-478. https://doi.org/10.5216/ ia.v39i3.27535

Guarín, M. R., Malagón, N. R., Gempeler, J. G., \& Garzón, D. F. (2013). Factores asociados con intento de suicidio y comportamientos de autolesión no suicida en pacientes con trastornos del comportamiento alimentario. Revista Colombiana de Psiquiatría, 43(supl. 1), 19-26. https://doi. org/10.1016/j.rcp.2013.11.007

Hambleton, R., \& Jones, R. (1993). Comparison of classical test theory and item response theory and their applications to test development. Education of Measure, 12(3), 38-47. https://doi.org/10.1111/j.1745-3992.1993.tb00543.x

Jiménez-Flores, P., Jiménez-Cruz, A., \& Bacardí-Gascón, M. (2017). Insatisfacción con la imagen corporal en niños y adolescentes: revisión sistemática. Nutrición Hospitalaria, 34(2), 479-489. http://dx.doi.org/10.20960/nh.455

Leins, J., Waldorf, M., Kollei, I., Rinck, M., \& Loeber, S. (2018). Approach and avoidance: Relations with the thin body ideal in women with disordered eating behavior. Psychiatry Research, 269, 286-292. https://doi. org/10.1016/j.psychres.2018.08.029

Linacre, J. M. (2002). What do Infit and Outfit, Mean-square and Standardized mean? Rasch Measurement Transaction, 168, 878. http://www.Rasch.org/rmt/rmt162f.htm

Linardon, J., Kothe, E. J., \& Fuller-Tyszkiewicz, M. (2019). Efficacy of psychotherapy for bulimia nervosa and bingeeating disorder on self-esteem improvement: Meta-analysis. 
European Eating Disorders Review, 27(2), 109-123. https:// doi.org/10.1002/erv.2662

Llorente, E., Gleaves, D. H., Warren, C. S., Pérez-de-Eulate, L., \& Rakhkovskaya, L. (2015). Translation and validation of a spanish version of the sociocultural attitudes towards appearance questionnaire-4 (SATAQ-4). International Journal of Eating Disorders, 48(2), 170-175. https://doi. org/10.1002/eat.22263

Londoño. P. C., Velasco, S. M., Pardo, C. A., Martínez, M. P., Pulido, Y. Y., \& Pareja, L. F. (2019). Condiciones psicométricas de la Escala de Acontecimientos Vitales Estresantes usada en población colombiana. Actualidades en Psicología, 33(126), 83-96. https://doi.org/10.15517/ AP.V33I126.30158

Meléndez, J. A., Bustinzar, A. R., de la Vega Morales, R. I., Chávez-Peón, P. B., \& Moreno, L. R. (2017). Validation of the Hospital Anxiety and Depression Scale in Mexican population with eating disorders. Revista Mexicana de Trastornos Alimentarios, 8(2), 123-130. http://dx.doi. org/10.1016/j.rmta.2017.05.001

Montero, I., \& León, O. G. (2002). Clasificación y descripción de las metodologías de investigación en Psicología. International Journal of Clinical and Health Psychology 2(3), 503-508. https://www.redalyc.org/pdf/337/33720308. pdf/

Murawski, B., Elizathe, L., Custodio, J., \& Rutsztein, G. (2015). Argentine validation of the Sociocultural Attitudes Towards Appearance Questionnaire-3. Mexican Journal of Eating Disorders, 6(2), 73-90. http://dx.doi.org/10.1016/j. rmta.2015.09.001

Nunnally, J. C., \& Bernstein, I. H. (1978). Psychometric testing. McGraw-Hill.

Ochoa, N. C., Ramos, E. C., Méndez, A. J., \& Alamilla, E. K. (2013). Prevalencia de trastornos de la alimentación en adolescentes. Revista de Especialidades Médico-Quirúrgicas, 18(1), 51-55. https:/www.medigraphic.com/cgi-bin/new/ resumen.cgi?IDARTICULO $=41342$

Organización Mundial de la Salud (OMS). (2018). Desarrollo en la adolescencia. http://www.who.int/ maternal_child_adolescent/topics/adolescence/dev/es/

Paniagua, R. (2015). Metodología para la validación de una escala o instrumento de medida. [Artículo en línea]. Universidad de Antioquia, Medellín. http://www.udea. edu.co/wps/wcm/connect/udea/d76a0609-c62d-4dfb$83 \mathrm{dc}-5313 \mathrm{c} 2 \mathrm{aed} 2 \mathrm{f} 6 / \mathrm{METODOLOG} \% \mathrm{C} 3 \% 8 \mathrm{DA}+\mathrm{PAR}$ $\mathrm{A}+\mathrm{LA}+\mathrm{VALIDACI} \% \mathrm{C} 3 \% 93 \mathrm{~N}+\mathrm{DE}+\mathrm{UNA}+\mathrm{ESCALA}$. pdf?MOD=AJPERES

Pardo, C., \& Rocha, M. (2010). Compendio de los manuales del SERCE. Manual para el procesamiento y análisis de datos aplicación piloto. OREALC.

Pedrosa, I., Suárez-Álvarez, J., \& García-Cueto, E. (2013). Evidencias sobre la validez de contenido: avances teóricos y métodos para su estimación. Acción psicológica, 10(2), 3-18. http://dx.doi.org/10.5944/ap.10.2.11820
Pejenaute, E. G., \& Labari, M. E. (2018). Vigorexia. Formación Médica Continuada en Atención Primaria, 25(5), 262-269. https://doi.org/10.1016/j.fmc.2017.08.003

Pérez, M. B., Nieto, A. C., Palacio, L., \& Majul, F. (2015). Bulimia nerviosa y factores de riesgo asociados en adolescentes escolarizados de 14 a 18 años en Barranquilla (Colombia). Salud Uninorte, 31(1), 36-52. https://www. redalyc.org/pdf/817/81739659005.pdf

Pérez, S., Marco, J. H., \& Cañabate, M. (2018). Nonsuicidal self-injury in patients with eating disorders: prevalence, forms, functions, and body image correlates. Comprehensive Psychiatry, 84, 32-38. https://doi. org/10.1016/j.comppsych.2018.04.003

Plumed, J., Gimeno, N., Barberá, M., Ruiz, E., Conesa, L., Rojo-Bofill, L. M., \& Rojo, L. (2019). Burlas como factor de riesgo para conductas alimentarias anómalas: estudio prospectivo en una población adolescente. Revista de Psiquiatría y Salud Mental, 12(1), 17-27. https://doi. org/10.1016/j.rpsm.2017.06.007

Prnjak, K., Pemberton, S., Helms, E., \& Phillips, J. G. (2019). Reactions to ideal body shapes. The Journal of General Psychology, 147(4), 361-380. https://doi.org/10.1080/0022 1309.2019.1676190

Ralph-Nearman, C., \& Filik, R. (2020). Development and validation of new figural scales for female body dissatisfaction assessment on two dimensions: thin-ideal and muscularity-ideal. BMC Public Health, 20(1) 1-11. https://doi. org/10.1186/s12889-020-09094-6

Rheanna, N., Thompson, J. K., \& Brent. J. (2013). Effects of exposure to thin-ideal media images on body dissatisfaction: Testing the inclusion of a disclaimer versus warning label. Body Image, 10(4), 472-480. https://doi.org/10.1016/j. bodyim.2013.04.004

Rodgers, R. F., Lowy, A. S., Halperin, D. M., \& Franko, D. L. (2016). A Meta-Analysis Examining the Influence of Pro-Eating Disorder Websites on Body Image and Eating Pathology. European Eating Disorders Review, 24(1), 3-8. https://doi.org/10.1002/erv.2390

Rodgers, R. F., Schaefer, L. M., Thompson, J. K., Girard, M., Bertrand, M., \& Chabrol, H. (2016). Psychometric properties of the Sociocultural Attitudes Towards Appearance Questionnaire-4 (SATAQ-4) in French women and men. Body Image, 17, 143-151. https://doi.org/10.1016/j. bodyim.2016.03.002

Ruiz, A. O., Vázquez, R., Mancilla, J. M., \& Trujillo, E. M. (2010). Factores socioculturales de hijas y padres en los trastornos del comportamiento alimentario. Enseñanza $e$ Investigación en Psicología, 15(2), 311-324. https://www. redalyc.org/pdf/292/29215980005.pdf/

Salmeron, M. A., Román, C., \& Casas, J. (2017). Trastornos del comportamiento alimentario. Pediatría Integral, XXI(2), 82-91. https://www.pediatriaintegral.es/publicacion-201703/trastornos-del-comportamiento-alimentario-2/ 
Schaefer, L. M., Burke, N. L., Thompson, J. K., Dedrick, R. F., Heinberg, L. J., Calogero, R. M., Bardone-Cone, A. M., Higgins, M. K., Frederick, D. A., Kelly, M., Anderson, D. A., Schaumberg, K., Nerini, A., Stefanile, C., Dittmar, H., Clark, E., Adams, Z., Macwana, S., Klump, K. L., ... Swami, V. (2015). Development and validation of the Sociocultural Attitudes Towards Appearance Questionnaire-4 (SATAQ-4). Psychological Assessment, 27(1), 54-67. https://doi.org/10.1037/a0037917

Schaefer, L. M., Thompson, J. K., Heinberg, L. J., Calogero, R. M., Nerini, A., Dittmar, H., ... Macwana, S. P. (2012, May). Validation of the Sociocultural Attitudes Towards Appearance Questionnaire-4 (SATAQ-4) in Italian, British, and Australian Women [Poster presented at The International Conference on Eating Disorders]. University of South Florida. https://bit.ly/2RYwo7D

Schnettler, B., Miranda-Zapata, E., Grunert, K. G., Lobos, G., Denegri, M., Hueche, C., \& Poblete, H. (2018). University student profiles according to satisfaction with life, food and family. Revista Chilena De Nutricion, 45(3), 263-270. http://dx.doi.org/10.4067/s0717-75182018000400263

Shahyad, S., Pakdaman, S., Shokri, O., \& Saadat, S. H. (2018). The role of individual and social variables in predicting body dissatisfaction and eating disorder symptoms among iranian adolescent girls: an expanding of the tripartite influence mode. European Journal of Translational Myology, 28(1), 99-104. https://doi.org/10.4081/ejtm.2018.7277

Sharpe, H., Griffiths, S., Choo, T. H., Eisenberg, M. E., Mitchison, D., Wall, M., \& Neumark-Sztainer, D. (2018). The relative importance of dissatisfaction, overvaluation and preoccupation with weight and shape for predicting onset of disordered eating behaviors and depressive symptoms over 15 years. International Journal of Eating Disorders, 51(10), 1168-1175. https://doi.org/10.1002/eat.22936

Solis, S. V., Perez, R. G., \& Sanchez-Carracedo, D. (2019). Frecuencia de comidas en familia y riesgo de trastornos de la conducta alimentaria en adolescentes de España y Perú. Revista Latinoamericana de Psicología, 51(1), 48-58. https://doi.org/10.14349/rlp.2019.v51.n1.6
Thompson, J. K., Van Den Berg, P., Roehrig, M., Guarda, A. S., \& Heinberg, L. J. (2004). The sociocultural attitudes towards appearance scale-3 (SATAQ-3): Development and validation. International Journal of Eating Disorders, 35(3), 293-304. https://doi.org/10.1002/eat.10257

Uchôa, F. N., Uchôa, N. M., Daniele, T. M. da C., Lustosa, R. P., Garrido, N. D., Deana, N. F., Alves, N. (2019). Influence of the mass media and body dissatisfaction on the risk in adolescents of developing eating disorders. International Journal of Environmental Research and Public Health, 16(9), 1508. https://doi.org/10.3390/ijerph16091508

Vaquero-Cristóbal, R., Alacid, F., Muyor, J. M., \& LópezMiñarro, P. Á. (2013). Imagen corporal: revisión bibliográfica. Nutrición Hospitalaria, 28(1), 27-35. http://dx.doi. org/10.3305/nh.2013.28.1.6016

Vázquez, R., Álvarez, G., \& Mancilla, J.M. (2000). Consistencia interna y estructura factorial del cuestionario de influencia de los modelos estéticos corporales (CIMEC), en población mexicana. Salud Mental, 23(6), 1824. https://www.medigraphic.com/cgi-bin/new/resumenI. cgi?IDARTICULO=22624

Williams-Kerver, G. A., \& Crowther, J. H. (2020). Emotion differentiation and disordered eating behaviors: The role of appearance schemas. Eating Behaviors, 37, 101369101377. https://doi.org/10.1016/j.eatbeh.2020.101369

Yamamiya, Y., Shimai, S., Schaefer, L. M., Thompson, J. K., Shroff, H., Sharma, R., \& Ordaz, D. L. (2016). Psychometric properties and validation of the Sociocultural Attitudes Towards Appearance Questionnaire-4 (SATAQ-4) with a sample of Japanese adolescent girls. Body Image, 19, 8997. https://doi.org/10.1016/j.bodyim.2016.08.006

Yamamiya, Y., Shroff, H., Schaefer, L. M., Thompson, J. K., Shimai, S., \& Ordaz, D. L. (2019). An exploration of the psychometric properties of the SATAQ-4 among adolescent boys in Japan. Eating Behaviors, 32, 31-36. https://doi. org/10.1016/j.eatbeh.2018.12.001 